\title{
The spectrum of polypoidal choroidal vasculopathy in Caucasians: clinical characteristics and proposal of a classification
}

\author{
Elon H. C. van Dijk ${ }^{1}$ • Danial Mohabati ${ }^{1}$ • Simona Veselinovic ${ }^{1}$ • Wing H. Chung ${ }^{1}$ - Greet Dijkman ${ }^{1} \cdot$ Camiel J. F. Boon $^{1,2,3}$
}

Received: 19 March 2020 / Revised: 6 July 2020 / Accepted: 10 July 2020 / Published online: 18 August 2020

(C) The Author(s) 2020

\begin{abstract}
Purpose To describe the clinical characteristics and outcome of polypoidal choroidal vasculopathy (PCV), also known as aneurysmal type 1 (sub-retinal pigment epithelium (RPE)) neovascularization, in Caucasian patients.

Methods Single-centre study in 66 Caucasian patients with a diagnosis of PCV based on optical coherence tomography scan and indocyanine green angiography. Clinical characteristics and multimodal imaging were collected and assessed by an experienced retina specialist. Results This study involved 74 eyes of 66 patients with PCV, with a mean age at onset of 73 years and a female preponderance of $66 \%$. The mean number of polypoidal lesions per eye was 1 (range: 1-5 lesions), out of which $75 \%$ was located in the macula and $19 \%$ in the peripapillary region. Of the 74 eyes, 37 eyes (50\%) had PCV associated with a drusenoidal neovascular age-related macular degeneration (AMD) phenotype (PCV-AMD) and 18 eyes (24\%) had PCV associated with non-polypoidal type 1 choroidal neovascularization/branching vascular network (PCV-BVN) without signs of drusenoidal AMD, while 19 eyes (26\%) had idiopathic, isolated PCV (iPCV). The mean subfoveal choroidal thickness measured in 22 patients was $245 \mu \mathrm{m}$ (range: $71-420 \mu \mathrm{m}$ ). In $51 \%$ of patients, the initially performed therapy showed good anatomical recovery (resolution of intra- and subretinal fluid).

Conclusions A spectrum of PCV (aneurysmal type 1/sub-RPE neovascularization) can be seen in Caucasian patients. PCV associated with a drusenoidal neovascular AMD phenotype in Caucasians is phenotypically and presumably pathophysiologically more associated with neovascular AMD (PCV-AMD: type A PCV). However, this may not be the case for patients with PCV with non-polypoidal type 1 choroidal neovascularization or BVN and no signs of drusenoidal AMD (PCV-BVN: type B PCV), and for patients with idiopathic PCV without associated drusen or BVN (iPCV; type C PCV). Most patients have a thin choroid, even when drusen are absent. For the entire patient group, a moderate anatomical recovery was observed after treatment.
\end{abstract}

Keywords Aneurysmal type 1 neovascularization · Caucasians $\cdot$ Classification $\cdot$ Clinical characteristics $\cdot$ Polypoidal choroidal vasculopathy $\cdot$ Sub-retinal pigment epithelium neovascularization

Elon H. C. van Dijk and Danial Mohabati contributed equally to this manuscript, and have to be considered joint first authors.

Electronic supplementary material The online version of this article (https://doi.org/10.1007/s00417-020-04844-z) contains supplementary material, which is available to authorized users.

Camiel J. F. Boon

c.j.f.boon@lumc.nl

1 Department of Ophthalmology, Leiden University Medical Center, Albinusdreef 2, 2333 ZA Leiden, The Netherlands

2 Department of Ophthalmology, Amsterdam University Medical Centers, University of Amsterdam, Amsterdam, The Netherlands

3 Department of Ophthalmology, Leiden University Medical Center, P.O. Box 9600, 2300 RC Leiden, The Netherlands

\section{Introduction}

Idiopathic polypoidal choroidal vasculopathy (PCV) was first described in 1990 by Yannuzzi et al. as peculiar polypoidal subretinal lesions that were associated with haemorrhagic detachments of the retinal pigment epithelium (RPE) [1]. Later, Yannuzzi suggested that PCV may be a self-contained clinical entity, which involves elevated reddish to orange lesions on fundus examination, dilated inner choroidal vessels, and polypoidal vascular structures beneath a RPE detachment [2].

Recently, controversy has arisen regarding the definition of $\mathrm{PCV}$ and its association with neovascular age-related macular degeneration (nAMD) [2-5]. PCV and nAMD have been found to have similar environmental risk factors and to share molecular and genetic determinants involving the complement pathway [6]. Furthermore, several studies have shown 
that polypoidal lesions in PCV are often associated with a branching vascular network (BVN) of subretinal neovascularization between the RPE and Bruch's membrane, indicating that PCV could be a variant of type 1 neovascularization of any origin $[4,7]$. Patients with type 1 (sub-RPE) subretinal neovascularization can have an aneurysmal dilation (the "polyp'), often at the edge of the neovascularization. This aneurysmal dilation is also known with the term PCV, which strictly speaking is a misnomer because we have learned that neovascular lesions that characterize PCV do not arise directly from the choroid [3]. The aneurysmal dilation or PCV typically shows as a well-circumscribed hyperfluorescent lesion already in the early phase on indocyanine green angiography (ICGA). The non-aneurysmal part of the type 1 neovascular lesion is often referred to as a BVN, which can therefore be present in every macular neovascular condition $[3,4,6]$. In comparison with nAMD without PCV, patients with PCV may show relative resistance to anti-vascular endothelial growth factor (VEGF) treatment $[8,9]$. Based on imaging studies, some authors have suggested that the term PCV should be reserved for patients in whom findings characteristic for AMD, such as drusen, pigmentary changes, and geographic atrophy, are absent $[7,10,11]$.

PCV has been studied mostly in Asian populations. These studies have shown a relatively high prevalence of PCV of up to $61.6 \%$ in Asian patients who presumably had nAMD [10, 12, 13]. However, this prevalence has been shown to be only $8.7 \%$ in Caucasian patients with a suspicion of nAMD [14, 15], although a recent study described a prevalence of up to $31.9 \%$ [16]. A study in which the occurrence of PCV was compared directly between an Asian and non-Asian nAMD cohort showed a large difference: $48 \%$ of Japanese patients and 9\% of French nAMD patients had PCV [17]. Despite the fact that drusen have traditionally been considered hallmark and even mandatory lesions for the diagnosis of AMD [18], we have also described a spectrum of nAMD in elderly Caucasian patients without drusen in the fellow eye, in whom 5 patients $(10 \%)$ were shown to have evidence of PCV in the affected eye [19]. This may indicate that the aetiology of non-drusen-associated nAMD and PCV may be different from drusenoidal neovascular subtypes [19]. Little is known about the risk factors, clinical characteristics, and treatment outcome of PCV in Caucasians, as previously conducted studies had small sample sizes [20-23].

The purpose of this study is to describe the anatomical characteristics and the clinical course of PCV in the largest Caucasian population published so far. We show that there are different clinical manifestations of PCV in Caucasians, and propose a PCV classification into 3 subtypes, comparing our findings in the present study population to the available data on Caucasian nAMD patients, and to previously published characteristics of Asian PCV patients.

\section{Methods}

\section{Study population}

A retrospective review of medical charts was performed for patients who visited the Department of Ophthalmology of Leiden University Medical Center (Leiden, the Netherlands), a tertiary referral centre for medical retina diseases in The Netherlands. This study adhered to the tenets of the Declaration of Helsinki and all federal laws in The Netherlands, and approval for this retrospective study was obtained from the Medical Ethical Committee of Leiden University Medical Center. From 2000 to 2019, characteristics and multimodal imaging from consecutive patients who were suspected to have PCV were collected, based on the following: (1) a pink-orange subretinal nodular lesion on fundoscopy; (2) a peaked/dome-shaped elevation of the RPE, often with a hyperreflective subretinal accumulation beneath it; (3) non-response or only partial response to anti-VEGF treatment in patients with macular neovascularization without evidence of another diagnosis than AMD. All images were obtained from routine eye examinations and included colour fundus photographs (Topcon Corp., Tokyo, Japan), optical coherence tomography (OCT) scans with either the timedomain (Cirrus OCT Carl Zeiss Meditec, Dublin, CA, USA) or spectral-domain OCT (SD-OCT) (Spectralis HRA+OCT, Heidelberg Engineering GmbH, Heidelberg, Germany) device, ICGA (Spectralis HRA+OCT), and fluorescein angiography (FA) (performed with either the Topcon fundus camera or the Spectralis HRA+OCT camera).

Only patients of Caucasian ethnicity were included in the study if they met all of the following inclusion criteria: (1) a diagnosis of PCV was established when single or multiple focal nodular areas of hyperfluorescence were visible within the first 6 min after dye injection on ICGA, with or without a choroidal interconnecting vascular network, together with characteristic nodular hyperfluorescent structures on FA [3, 7, 15, 24-26]; (2) availability of clinical information and imaging for at least 1 visit after initial diagnosis and treatment (Fig. 1). Exclusion criteria were any of the following: (1) insufficient clinical data at diagnosis; (2) insufficient quality of multimodal imaging; (3) evidence of diagnoses other than either AMD or idiopathic PCV, with a possible pathophysiological background for PCV, such as central serous chorioretinopathy, as we aimed to include only patients who had PCV as 'primary disease'.

\section{Clinical examinations and classification}

Patients who met the inclusion criteria were graded in 3 subtypes of PCV based on the presence of drusen, CNV, and BVN on multimodal imaging which was assessed by an experienced retina specialist (CJFB). Since no clear definition 
for BVN was found in literature, we defined this as a network on ICGA within the first $6 \mathrm{~min}$ after injection of indocyanine green that corresponded to a 'double-layer sign' on OCT, corresponding to a shallow RPE detachment separated from Bruch's membrane by an area of moderate reflectivity $[27,28]$, without the presence of extensive (late) leakage on FA [7]. This is in contrast to the peaked/dome-shaped/'thumb-like' polyp [6]. On ICGA, a non-polypoidal type 1 neovascular lesion does not show early well-circumscribed hyperfluorescence, which is in contrast to a polypoidal lesion [6].

The cases were subsequently divided in 3 groups, based on the previous description of Coscas et al. [7], taking into account that a CNV or a BVN could also occur in patients in whom no signs of AMD are present. These 3 groups included the following: (1) PCV within the context of nAMD with the presence of $\geq 5$ drusen with a diameter of $\geq 63 \mu \mathrm{m}$ in the affected or fellow eye; (2) PCV in combination with either a non-polypoidal type $1 \mathrm{CNV}$ or BVN without signs of AMD (i.e. the presence of $<5$ either hard or soft drusen with a diameter of $\geq 63 \mu \mathrm{m}$ ) in the affected or fellow eye; (3) idiopathic PCV without drusen in the affected or fellow eye, and without signs of either CNV or BVN in the affected eye.

The number, shape, and location of the polypoidal lesions were subsequently assessed. The following shapes were distinguished: isolated polypoidal lesions, string-like polypoidal lesions (at least 3 solitary polyps in line) [29], and a cluster of grape-like polypoidal lesions [30]. The presence and location of polypoidal lesions were determined on ICGA imaging within $6 \mathrm{~min}$ after injection of indocyanine green [25]. After determination of the exact location of the fovea using SD-OCT and fundus photography, ICGA images were placed on top of the fundus photograph and an Early Treatment of Diabetic Retinopathy Study (ETDRS) grid with 3 solid circles of sizes 7200, 3600, and $1200 \mu \mathrm{m}$ was added. In those cases in which SD-OCT was not available, the location of the fovea was estimated using the timedomain OCT. The presence of polypoidal lesions for each anatomical region is depicted in Fig. 2.

For both eyes, information on the presence of macular haemorrhages and retinal atrophy were obtained at baseline. The origin of polypoidal lesions was studied by analysing possible interruptions of Bruch's membrane in those patients for whom SD-OCT of sufficient quality was available. Pachyvessels (pathologically dilated Haller layer with attenuation and thinning of the choriocapillaris and Sattle layer), and subfoveal choroidal thickness (distance from the outer part of the hyperreflective RPE layer to the hyperreflective line of the inner surface of the sclera), were studied in patients for whom OCT imaging of sufficient quality was available [3]. A subfoveal choroidal thickness of $>350 \mu \mathrm{m}$ was considered to be a pachychoroid in our relatively old patient group [31].

\section{Data collection at baseline and follow-up}

The following information was obtained for the visit at which PCV was diagnosed (baseline): age at onset, best-corrected visual acuity (BCVA; in Snellen letters, and subsequently converted to ETDRS letters), subjective symptoms before diagnosis of PCV, initial diagnosis at the referring ophthalmologist before suspicion of PCV at our centre, treatments before suspicion of PCV and after diagnosis of PCV, and total duration of follow-up. At the first follow-up visit and visits at on average at 6,12 , and 24 months after first treatment, the following information was obtained: BCVA, evaluation of subjective symptoms, performed treatments, treatment effect (defined as complete resolution of either intraretinal fluid or subretinal fluid) on OCT), and disease recurrence. Patients were noted as lost to follow-up, when no disease reoccurrence was observed at clinical evaluation in our centre after treatment and patient was thereafter sent back to the referring ophthalmologist.

\section{Statistical analysis}

Statistical analysis was performed using IBM SPSS Statistics, version 23 (IBM, Armonk, NY, USA). A one-way ANOVA test was performed for normal distributed numeric data, and both a Wilcoxon signed rank test and a Kruskal-Wallis test were used for not normally distributed numeric data. For the analysis of the categorical data, a chi-square test or a McNemar's test, or a Fisher's exact test was used. A $P$ value of $<0.05$ was considered statistically significant for all performed analyses.

\section{Results}

\section{Clinical characteristics}

A total of 74 eyes of 66 consecutive patients, with a mean age of 73 years (range: 51-90), with PCV were included in this study. In 44 of these eyes a pink-orange subretinal nodular lesion on fundoscopy, and/or a peaked/dome-shaped elevation of the RPE, usually with a hyperreflective subretinal accumulation beneath it, was found at baseline, whereas in 30 eyes a macular neovascularization without evidence of another diagnosis than AMD that either did not respond or only partially responded to previous anti-VEGF treatment was seen. The characteristics of the participants are summarized in Table 1. Table 1 also shows the BCVA of 3 different groups of PCV patients, for whom a follow-up of 24 months was available. At baseline, the median BCVA was 66.3 ETDRS letters and showed no differences between the 3 groups that comprised of a relatively low number of patients $(P=0.082)$. 


\section{Characteristics of polypoidal lesions}

In the 74 eyes, a total number of 144 polypoidal lesions could be identified (Table 2), which all could be found below the RPE, compatible with a type 1 neovascularization. Multiple lesions were seen in 30 eyes (41\%; range: 1 to 5 lesions; median: 1 lesion). Eighty-six of all polypoidal lesions (60\%) were solitary, $46(32 \%)$ were in cluster(s), and $10(7 \%)$ occurred in a string-like configuration (Fig. 2). Thirty-seven eyes (50\%) could be classified as having PCV within the context of nAMD, 18 eyes (24\%) as having PCV in combination with a non-polypoidal type $1 \mathrm{CNV}$ or BVN but without signs of AMD in both eyes, and 19 eyes (26\%) as having idiopathic polyps without either drusen in both eyes or signs of either CNV or BVN in the fellow eye (Fig. 1). The exact location of the polypoidal lesion was determined by using an SD-OCTguided grid, with which $75 \%$ of the 144 polypoidal lesions were located within the macular region. The distribution of polypoidal lesions is depicted in Fig. 2.

Since the majority of our patients have been included in the pre-(SD-)OCT era, an SD-OCT was available for only 23 eyes $(31 \%)$. However, in 6 cases it was not possible to analyse the interruptions of Bruch's membrane due to either masking shadowing effects caused by either overlying sub-RPE abnormalities (4 eyes) or an incomplete depiction of the polypoidal lesion on OCT (2 eyes). Three eyes (18\%) had an interruption of more than $30 \mu \mathrm{m}$, with apparent direct communication between the polypoidal lesion and the underlying choroidal vasculature. For 22 affected eyes, the subfoveal choroidal thickness could be studied on SD-OCT, showing a mean of $245 \mu \mathrm{m}$ (range: 71$420 \mu \mathrm{m})$. Despite the fact that most of these patients have a relatively thin choroid, (relative) pachyvessels were seen in 6 $(27.3 \%)$ of these eyes. Of the 21 patients with a subfoveal choroidal thickness $<350 \mu \mathrm{m}$, the presence or absence of drusen in the affected and fellow eye could be determined in 17 patients $(81.0 \%)$. Fifteen of these patients $(88.2 \%)$ did not have any drusen. One patient (5.9\%) had extensive reticular pseudodrusen in both eyes, and the second patient had large, confluent sub-RPE drusen in the fellow eye.

\section{Treatment outcome}

Thirty-five eyes out of 73 patients with available information (47\%) were treatment-naive at the moment of referral to our tertiary referral centre. Out of the 38 eyes $(51 \%)$ which received treatment before referral, 30 patients $(79 \%)$ had received intravitreal anti-VEGF injections; 4 patients had received a combination therapy of PDT and thermal laser (11\%); 2 patients $(5 \%)$ had been treated with a combination therapy of both PDT and intravitreal anti-VEGF injections, and thermal laser; and 2 patients $(5 \%)$ had been treated with a combination therapy of PDT and intravitreal anti-VEGF injections.
Performed treatments for PCV during follow-up are shown in Table 3. After the diagnosis of PCV was established, in this historical cohort thermal laser was performed in 25 patients (34\%), a combination of PDT and intravitreal injection with anti-VEGF in 25 patients (34\%), and 24 patients $(31 \%)$ received either other treatment combinations as initial therapy or patients received no treatment. Additional treatment was required in 29 patients (39\%) at first visit after initial therapy, in 17 patients (33\%) at 6 months after initial therapy, and in 10 patients (26\%) at 12 months after initial therapy, respectively. Intravitreal injection with anti-VEGF medication was the most frequently performed additional therapy (Supplementary Table 1).

\section{Discussion}

To the best of our knowledge, we describe the largest group of Caucasian PCV patients to date. We found a spectrum of $\mathrm{PCV}$, and propose a classification, based on a previous description of Coscas et al. [7]. In the current Caucasian patient cohort, we were able to distinguish 3 types of PCV: the first and largest group of patients had PCV within the context of typical drusen-associated nAMD, with the presence of $\geq 5$ drusen with a diameter of $\geq 63 \mu \mathrm{m}$ in the affected and fellow eye (type A PCV); the second group comprised patients with $\mathrm{PCV}$ in combination with a non-polypoidal type $1 \mathrm{CNV}$ or

Fig. 1 Multi-modal imaging of subtypes of polypoidal choroidal vasculopathy. A, multimodal imaging in a patient with polypoidal choroidal vasculopathy (PCV) associated with drusenoidal neovascular age-related macular degeneration (AMD). A1, colour fundus photography shows drusen, central hyperpigmentation, and an orange nodule superiorly in the macula. A2, late-phase fundus fluorescein angiography (FA) shows leakage of fluorescein in an occult neovascular pattern. A3, indocyanine green angiography (ICGA) shows a single solitary polypoidal lesion without a vascular network. A4, spectral-domain optical coherence tomography (SD-OCT) shows subretinal fluid and a peaked elevation of the retinal pigment epithelium (RPE) that represents the polypoidal lesion. $\mathrm{B}$, multimodal imaging in a patient with non-AMD associated $\mathrm{PCV}$ in combination with a branching vascular network (BVN). B1, colour fundus photography shows an orange nodule within the macula, without signs of AMD. B2, late-phase FA shows leakage of fluorescein that corresponds with the polypoidal lesion. B3, ICGA shows 3 solitary polypoidal lesions associated with a vascular network nasally. B4, SD-OCT showing a dome-shaped RPE detachment with a hyperreflective subretinal accumulation that corresponds with the polypoidal lesion. This polypoidal lesion is associated with a doublelayer sign on the nasal side, which represents a BVN. C, multimodal imaging in a patient with idiopathic PCV. C1, colour fundus photography showing peripapillary atrophy associated with a brown-reddish nodule within the papillomacular bundle. C2, Late-phase FA showing minimal leakage of fluorescein that corresponds with the polypoidal lesion and window defects that correspond with peripapillary atrophy. C3, ICGA shows a solitary polypoidal lesion without a vascular network. C4, On SD-OCT 2 dome-shaped RPE detachments with a hyperreflective subretinal accumulation that correspond with the polypoidal lesions, can be observed. 

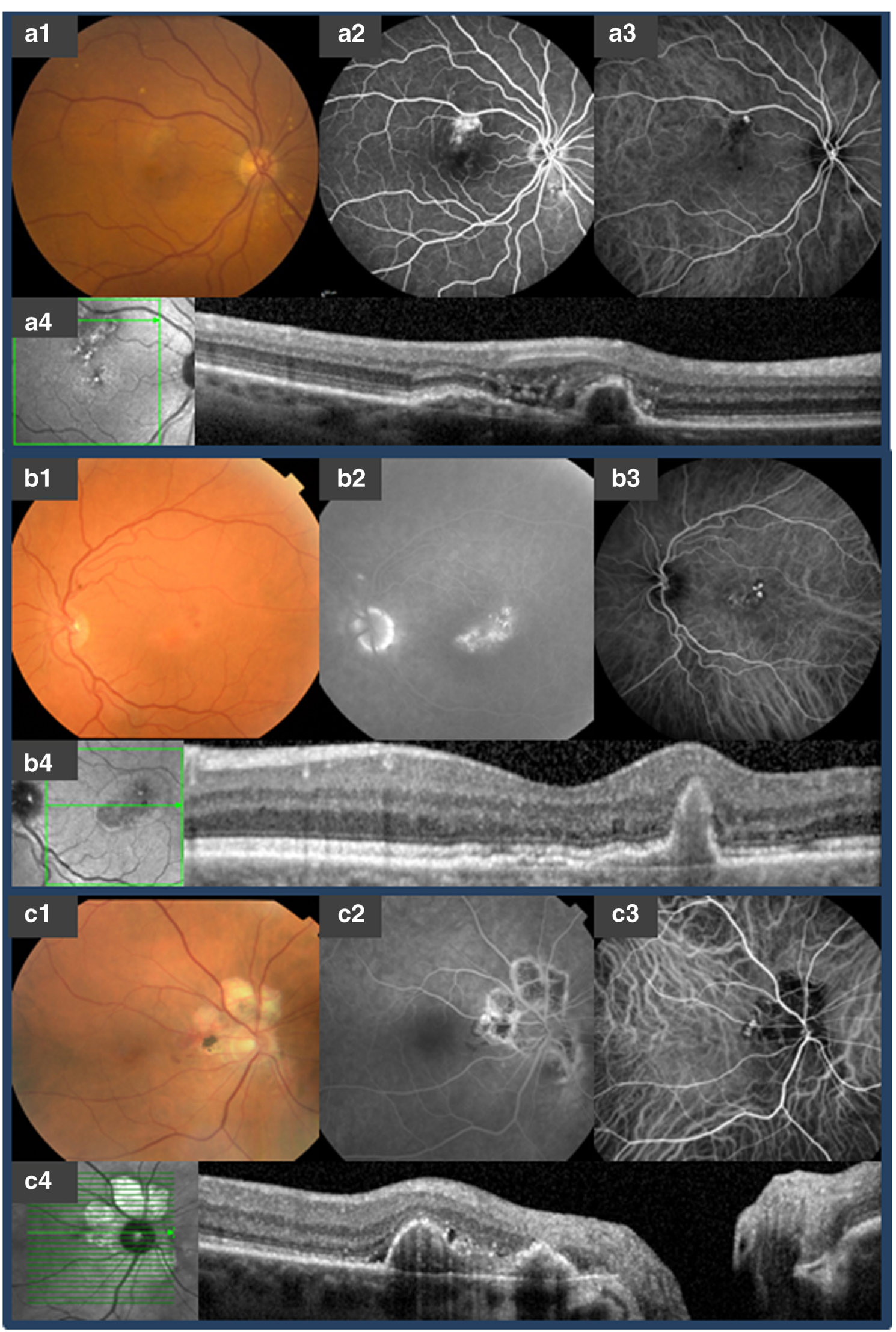


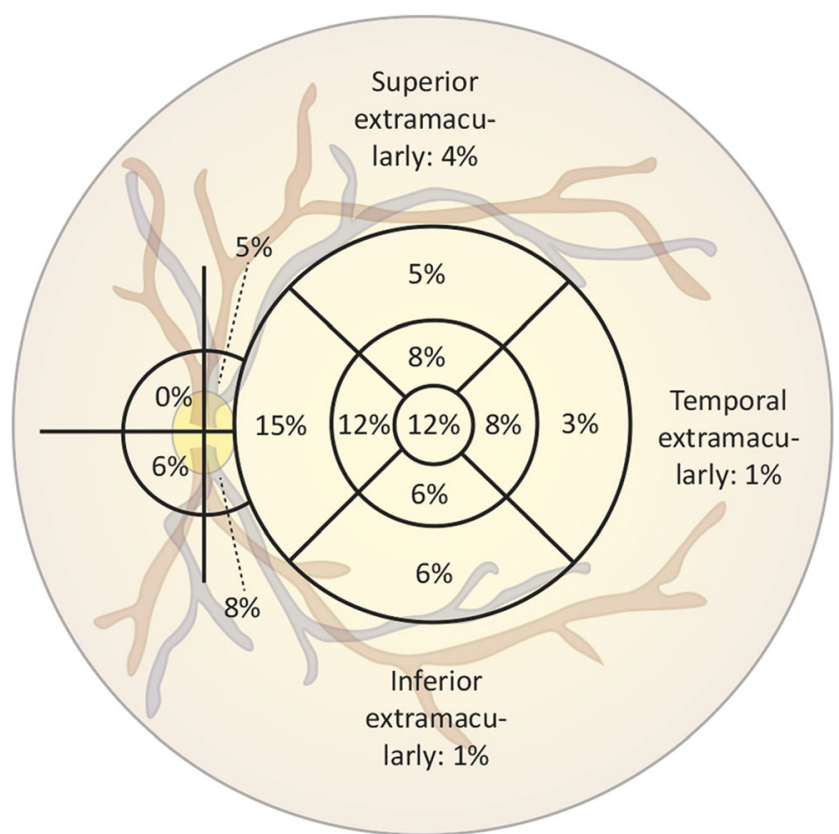

Fig. 2 Distribution of 144 polypoidal lesions among 74 eyes of Caucasian polypoidal choroidal vasculopathy patients

BVN component, but without drusen as typical signs of AMD (type B PCV); the third group included patients with idiopathic PCV with neither drusen nor signs of non-polypoidal type 1 $\mathrm{CNV}$ or BVN (also not in the fellow eye; type C PCV). While drusen-associated PCV type A generally has a normal to thin choroid without pachyvessels, types B and $\mathrm{C}$ may also have a pachychoroid in a considerable proportion (but not all) of the cases $[7,9]$. However, a remarkably high number of patients who received SD-OCT in our study had a thin choroid, also in cases of type B and C PCV.

Our study reveals additional differences between Caucasian and Asian PCV patients. PCV in our study tends to manifest at a slightly older age ( 73 years versus $65-73$ years in Asians) [12, 13, 32-35]. A male preponderance (42-83\%) is reported in Asians, while in the current cohort the opposite was found, with a female preponderance of $66 \%[12,13$, 32-35], which is also in contrast to some previous studies on PCV in Caucasians, in whom men and women were in general evenly represented $[8,36]$. PCV accounts for about $22-55 \%$ of cases manifesting as nAMD in Asians, compared with $8.7 \%$ of Caucasian population [15], but some authors have described a prevalence of PCV in Caucasians up to 31.9\%. Polypoidal lesions in Asians are often located in the macular region (78-94\%) rather than in the peripapillary region (6-14\%), while this difference is less pronounced in Caucasians (75\% in the macula and 19\% in the peripapillary region). [12, 13, 32, 33, 35] Most remarkably, half of the patients in the current Caucasian PCV study showed clinical similarities with nAMD in Caucasians, including the following: age [37, 38], laterality [33, 38], presence of drusen, and presence of a type 1 neovascularization $[39,40]$. This is in line with the increasingly established idea of PCV being a variant manifestation of a type 1 neovascularization within the spectrum of AMD $[4,11,15]$, which is also in line with findings

Table 1 Baseline characteristics of all Caucasian polypoidal choroidal vasculopathy patients

\begin{tabular}{|c|c|c|c|c|c|}
\hline & \multirow[t]{2}{*}{ Total population (eyes) } & \multicolumn{3}{|l|}{ Subtypes of PCV } & \multirow[t]{2}{*}{$P$ value } \\
\hline & & $\begin{array}{l}\text { PCV in context nAMD } \\
\text { (drusen; type A) }\end{array}$ & $\begin{array}{l}\text { PCV and non-polypoidal } \\
\text { type } 1 \mathrm{CNV} / \mathrm{BVN} \text { (type B) }\end{array}$ & $\begin{array}{l}\text { Idiopathic } \\
\text { PCV (type C) }\end{array}$ & \\
\hline Patients (eyes) & $66(74)$ & $33(37)$ & $17(18)$ & $16(19)$ & \\
\hline Mean age at onset in years (range) & $73(51-90)$ & $73(51-90)$ & $70(57-90)$ & $76(58-89)$ & $0.165^{\mathrm{a}}$ \\
\hline BCVA in ETDRS letters $( \pm$ SD) & $66.3(2.3)$ & $63.8(3.5)$ & $62.1(4.3)$ & $75.1(4.1)$ & $0.082^{\mathrm{a}}$ \\
\hline Male gender $(\%)$ & $25(34)$ & $11(30)$ & $9(50)$ & $5(26)$ & $0.239^{\mathrm{b}}$ \\
\hline \multicolumn{2}{|l|}{ Subjective symptoms (\%) } & & & & $0.292^{\mathrm{c}}$ \\
\hline Blurred vision & $36(48.6)$ & $22(59.5)$ & $8(44.4)$ & $6(31.6)$ & \\
\hline Spot in the field of vision & $10(13.5)$ & $2(5.4)$ & $4(22.2)$ & $4(21.1)$ & \\
\hline Metamorphopsia & $9(12.2)$ & $4(10.8)$ & $3(16.7)$ & $2(10.5)$ & \\
\hline Other & $18(24.3)$ & $8(21.6)$ & $3(16.7)$ & $7(36.8)$ & \\
\hline None & $1(1.4)$ & $1(2.7)$ & - & - & \\
\hline Median follow-up duration in days (range) & $343(14-1704)$ & $344(14-816)$ & $281(35-1704)$ & $349(38-840)$ & $0.919^{\mathrm{d}}$ \\
\hline
\end{tabular}

${ }^{a}$ One-way ANOVA

${ }^{\mathrm{b}} X^{2}$ test

${ }^{\mathrm{c}}$ Fisher's exact test

${ }^{\mathrm{d}}$ Kruskal-Wallis test

$B C V A$, best-corrected visual acuity; ETDRS, Early Treatment of Diabetic Retinopathy Study; $n$, number of eyes; $P C V$, polypoidal choroidal vasculopathy; $S D$, standard deviation 
Table 2 Clinical characteristics of all Caucasian polypoidal choroidal vasculopathy patients

\begin{tabular}{|c|c|c|c|c|c|}
\hline & $\begin{array}{l}\text { Total population } \\
\text { (eyes) }\end{array}$ & $\begin{array}{l}\text { PCV in context nAMD } \\
\text { (drusen; type A); } \\
n=37\end{array}$ & $\begin{array}{l}\text { PCV and non-polypoidal } \\
\text { type } 1 \mathrm{CNV} / \mathrm{BVN} \text { (type B); } \\
n=18\end{array}$ & $\begin{array}{l}\text { Idiopathic PCV } \\
\text { (type C); } \\
n=19\end{array}$ & $P$ value \\
\hline Number of polypoidal lesions, median (range) & $1(1-5)$ & $1(1-5)$ & $1(1-5)$ & $1(1-3)$ & $0.238^{\mathrm{a}}$ \\
\hline $\begin{array}{l}\text { Macular haemorrhage } \\
\text { Yes }\end{array}$ & $13(17.6)$ & $7(18.9)$ & $3(16.7)$ & $3(15.8)$ & $1.000^{\mathrm{b}}$ \\
\hline $\begin{array}{l}\text { Bilateral PCV, } n(\%) \\
\text { Yes }\end{array}$ & $16(21.6)$ & $8(21.6)$ & $2(11.1)$ & $6(31.6)$ & $0.637^{b}$ \\
\hline No & $51(68.9)$ & $26(70.3)$ & $14(77.8)$ & $11(57.9)$ & \\
\hline Unknown & $7(9.5)$ & $3(8.1)$ & $2(11.1)$ & $2(10.5)$ & \\
\hline
\end{tabular}

${ }^{\text {a }}$ Type 2 and type 3 neovascularization did not occur in this cohort

${ }^{\mathrm{b}}$ Fisher's exact test

$B V N$, branching vascular network; $C N V$, choroidal neovascularization; $n$, number of eyes; $n A M D$, neovascular age-related macular degeneration; $P C V$, polypoidal choroidal vasculopathy

from Balaratnasingam et al., who consider PCV/pachychoroid neovasculopathy to be part of the nAMD spectrum [3]. Remarkably, drusen have also been found to be present in $14.7-27 \%$ of PCV patients in previous studies [7, 15]. There is ample evidence that many, if not all, cases of PCV actually constitute an aneurysmal type 1 macular neovascularization, that is located between Bruch's membrane and the RPE, not in the choroid. [3, 4] However, this is still subject of controversy, and a recent expert panel effort could not reach consensus on the terminology of PCV versus aneurysmal type 1 neovascularization, because of differences in opinion on whether polypoidal structures represent 'simple' aneurysmal dilations or more complex structures [5]. Based on our findings on multimodal imaging, we also postulate that most cases of PCV are actually aneurysmal type 1 neovascularization. Still, in several patients in our study we had evidence of a potential connection of these lesions to the underlying choroid through an interrupted Bruch's membrane.

Traditionally, AMD by definition is associated with some kind of drusen, whether these are 'traditional' sub-RPE drusen and/or subretinal drusenoid deposits (also known as reticular pseudodrusen) [18, 41, 42]. However, we have previously shown that there is a spectrum of subretinal neovascularization in the macula in elderly without drusen in the affected eye as well as fellow eye [19]. In the current study on PCV, we had similar observations: $50 \%$ of PCV patients did not show drusen in the affected and fellow eye. Remarkably, $88.2 \%$ of patients with sufficiently gradable multimodal imaging had a choroidal thickness below $350 \mu \mathrm{m}$, without any drusen, and often without pachyvessels. This indicates that it is not uncommon for Caucasians to have PCV with a thin-to-normal choroidal thickness, and no drusen as signs of 'typical' AMD. Other studies on PCV in Caucasians have also described an absence of drusen in a considerable proportion of patients [7, 36]. The pathophysiology of these nAMD and PCV subtypes without drusen may therefore be different from drusenassociated AMD. For instance, pachychoroid neovasculopathy and pachychoroid-associated PCV may be pathophysiologically and clinically more close to central serous chorioretinopathy $[9,43]$. In our study, only 6 out of 22 patients with OCT imaging of sufficient quality showed pachyvessels. The mean subfoveal choroidal thickness in these patients was thin $(245 \mu \mathrm{m})$, and only 2 out of the 22 patients with SD-OCT measurements had a pachychoroid of $>350 \mu \mathrm{m}$ (396 and $420 \mu \mathrm{m}$, respectively) [31]. Therefore, we postulate that there is a third form of $\mathrm{PCV}$, which is associated with a relatively thin choroid, and is neither associated with drusenoid AMD, nor with pachychoroid and/or pachyvessels.

All PCV patients, regardless of whether there is a background of drusen or pachychoroid, had in common that they present with a neovascular and/or polypoidal degeneration at an age that is on average well over 70 ( 73 years in the current study). Hence, it seems rather arbitrary to exclude these forms of age-related neovascular degeneration of the macula without drusen (and without pachychoroid) from the definition of AMD. It is not always possible to assess whether these agerelated macular neovascularizations have a background of drusenoid AMD, pachychoroid, or neither of the aforementioned, while virtually all forms require some form of antiVEGF therapy. Thus, it may be reasonable to consider these 3 subtypes as different clinical manifestations of age-related macular neovascularization, or nAMD, when other causes such as myopic neovascularization and multifocal choroiditis have been excluded. However, we and others have hypothesised that the pathogenesis and genetic background of macular neovascularization associated with drusenoid AMD is different from that of for instance pachychoroidassociated macular neovascularization (pachychoroid neovasculopathy, with or without a polypoidal component) $[3,4,7,19]$. The clinical subtype of nAMD and PCV could 
Table 3 Performed treatments at different follow-up visits for all Caucasian polypoidal choroidal vasculopathy patients

\begin{tabular}{|c|c|c|c|c|c|}
\hline & Initial treatment $(n=74)$ & $\begin{array}{l}2 \text { months } \\
(n=74)\end{array}$ & 6 months $(n=51)$ & 12 months $(n=39)$ & Total treatment \\
\hline Anti-VEGF, $n(\%)$ & $2(2.7)$ & $15(20.3)$ & $9(17.6)$ & $5(12.8)$ & $2(2.7)$ \\
\hline Bevacizumab, $n$ (\# injections per eye) & $1(2)$ & $10(3)$ & $3(3)$ & $3(5)$ & $2(2)$ \\
\hline Ranibizumab, $n$ (\# injections per eye) & - & $3(2)$ & $5(3)$ & 1 (NA) & - \\
\hline Aflibercept, $n$ (\# injections per eye) & $1(2)$ & $1(2)$ & $1(6)$ & $1(3)$ & $1(2)$ \\
\hline $\mathrm{PDT}, n(\%)$ & $13(17.6)$ & $4(5.4)$ & $2(3.9)$ & - & $5(6.8)$ \\
\hline Normal settings PDT, $\mathrm{n}$ & 8 & 1 & 1 & & 2 \\
\hline Reduced settings PDT, $\mathrm{n}$ & 3 & 2 & 1 & & 1 \\
\hline Unknown settings PDT, $\mathrm{n}$ & 2 & 1 & - & & 2 \\
\hline Thermal laser, $n(\%)$ & $25(33.8)$ & $5(6.8)$ & $1(2)$ & $2(5.1)$ & $21(28.4)$ \\
\hline Anti-VEGF and PDT & $25(33.8)$ & $3(4.1)$ & $2(4.1)$ & $2(5.1)$ & $28(37.8)$ \\
\hline Bevacizumab, $n$ (\# injections per eye) & $20(2)$ & $1(2)$ & $2(2)$ & $2(2)$ & $32(2)$ \\
\hline Ranibizumab, $n$ (\# injections per eye) & $3(3)$ & 1 (NA) & - & - & $11(3)$ \\
\hline Aflibercept, $n$ (\# injections per eye) & $2(3)$ & 1 (NA) & - & - & $5(4)$ \\
\hline Normal settings PDT, $n$ & 21 & 2 & - & 1 & 25 \\
\hline Reduced settings PDT, $n$ & 4 & 1 & 1 & - & 9 \\
\hline Unknown settings PDT, $n$ & - & - & 1 & 1 & - \\
\hline Anti-VEGF and thermal laser, $n(\%)$ & $3(4.1)$ & $3(4.1)$ & $2(3.9)$ & $1(2.6)$ & $5(6.8)$ \\
\hline Bevacizumab, $n$ (\# injections per eye) & $2(2)$ & $3(4.3)$ & $2(4)$ & $1(3)$ & $9(2)$ \\
\hline Ranibizumab, $n$ (\# injections per eye) & $1(1)$ & - & - & & $1(1)$ \\
\hline Aflibercept, $n$ (\# injections per eye) & - & - & - & & - \\
\hline PDT and thermal laser, $n(\%)$ & $2(2.7)$ & - & - & - & $5(6.8)$ \\
\hline Normal settings PDT, $n$ & 1 & & & & 5 \\
\hline Reduced settings PDT, $n$ & - & & & & 1 \\
\hline Unknown settings PDT, $n$ & 1 & & & & 1 \\
\hline Anti-VEGF and PDT and thermal laser, $n(\%)$ & - & - & $1(2)$ & - & $6(8.1)$ \\
\hline Bevacizumab, $n$ (\# injections per eye) & & & $1(3)$ & & $8(3)$ \\
\hline Ranibizumab, $n$ (\# injections per eye) & & & - & & $2(2)$ \\
\hline Aflibercept, $n$ (\# injections per eye) & & & - & & $1(2)$ \\
\hline Normal settings PDT, $n$ & & & 1 & & 6 \\
\hline Reduced settings PDT, $n$ & & & - & & 1 \\
\hline Unknown settings PDT, $n$ & & & - & & 2 \\
\hline None, $n(\%)$ & $4(5.4)$ & $45(60.8)$ & $34(66.7)$ & $29(74.4)$ & $2(2.7)$ \\
\hline
\end{tabular}

$B C V A$, best-corrected visual acuity; $F U$, follow-up; $n$, number of eyes; $N A$, unknown/not available; $P D T$, photodynamic therapy; $V E G F$, vascular endothelial growth factor; \#, number

have therapeutic consequences: for instance, patients with PCV have been described to have a higher likelihood of resistance to anti-VEGF treatment in some studies $[8,9]$.

We have previously found differences in complement levels and genetic risk factors between central serous chorioretinopathy and AMD $[44,45]$. In an Asian population, single nucleotide polymorphisms in chromosomal regions of the complement factor $H$, age-related maculopathy susceptibility 2 (ARMS2), and high-temperature requirement factor Al genes have been associated both with PCV and nAMD [46]. However, an association between the rs10490924 variant of the ARMS2 gene and PCV in combination with a BVN was recently found, suggesting that $\mathrm{PCV}$ in combination with BVN shares genetic features with nAMD, which is in contrast with PCV without BVN [47, 48]. Little is known on genetic associations in Caucasian PCV patients [15]. While there is clinical and genetic evidence of common disease determinants and pathways between PCV and AMD, we hypothesise that these disease risk factors and pathways may diverge considerably between drusen-associated and non-drusen-associated PCV. Drusen have been described to be a hallmark of complement activation, whereas other factors such as a pachychoroid background may be more important in nondrusen-associated PCV [49]. Likewise, there may be 
significant differences in (patho)genetic background between PCV associated with drusen, PCV with nonpolypoidal type $1 \mathrm{CNV}$ or $\mathrm{BVN}$ but without drusen, and idiopathic solitary PCV without drusen.

In the present analysis of Caucasian PCV cases, we observed a good anatomical response to treatment. Here, the most frequently performed initial therapy was conventional thermal laser (36\%), closely followed by combination therapy of PDT and anti-VEGF injections (33\%), and PDT monotherapy (17\%). In $51 \%$ of patients, the initially performed therapy showed good anatomical recovery (Table 3, Supplementary Table 3). However, our study has several limitations, inherent to the fact that this was a retrospective study that also included follow-up of PCV patients who were initially phenotyped before the advent of novel imaging modalities such as SD-OCT. It should be noted that no firm conclusions on clinical outcome can be drawn from this analysis, given the inclusion bias (conventional laser is only used in extramacular polypoidal lesions, and nowadays performed only rarely), and the retrospective nature of this study. Due to the relatively small number of included patients, possible differences between both treatment regimens and between disease subtypes could not be studied. Moreover, choroidal thickness should be taken into account in more detail in the future, since OCT scans of sufficient quality were only available for a minority of our patients, also because of the fact that the majority of our patients have been included in the pre-(SD-)OCT era. OCT angiography could also provide additional information on the neovascular component of lesions to be studied. However, currently available devices would not be optimal to assess the PCV component, since direction of flow and low flow phenomena cannot be determined reliably [50]. Assessing pachyvessels in our study also proved to be challenging, without both the availability of a clear cut-off value for the definition of pachyvessels, and the difficulty of distinguishing several choroidal layers in patients with a relatively thin choroid [3].

Collectively, these findings support the hypothesis that PCV in Caucasians is phenotypically and presumably pathophysiologically more associated with AMD than in Asians with PCV, in whom a pachychoroid background is more common [7-9]. In Caucasian PCV, a spectrum of 3 subtypes of PCV can be distinguished (A, B, and C), out of which only type A PCV has a background of typical, drusen-associated AMD. Future prospective studies should aim at unravelling the underlying (patho)genetic background and optimal treatment in these 3 subgroups of PCV in the Caucasian population.

Funding information This research was supported by the Mathilde Grant, Stichting Leids Oogheelkundig Ondersteuningsfonds, Rotterdamse Stichting Blindenbelangen, Stichting Blindenhulp, and the Netherlands Organisation for Scientific Research (NWO). The funding organizations MD Fonds, Landelijke Stichting voor Blinden en Slechtzienden, Retina Netherlands, and BlindenPenning contributed through UitZicht. The aforementioned funding sources provided unrestricted grants and had no role in the design or conduct of this research.

\section{Compliance with ethical standards}

All procedures performed were in accordance with the ethical standards of the institutional and/or national research committee and with the 1964 Helsinki declaration and its later amendments or comparable ethical standards. Informed consent was obtained from all individual participants included in the study.

Conflict of interest The authors declare that they have no conflict of interest.

Open Access This article is licensed under a Creative Commons Attribution 4.0 International License, which permits use, sharing, adaptation, distribution and reproduction in any medium or format, as long as you give appropriate credit to the original author(s) and the source, provide a link to the Creative Commons licence, and indicate if changes were made. The images or other third party material in this article are included in the article's Creative Commons licence, unless indicated otherwise in a credit line to the material. If material is not included in the article's Creative Commons licence and your intended use is not permitted by statutory regulation or exceeds the permitted use, you will need to obtain permission directly from the copyright holder. To view a copy of this licence, visit http://creativecommons.org/licenses/by/4.0/.

\section{References}

1. Yannuzzi LA, Sorenson J, Spaide RF, Lipson B (1990) Idiopathic polypoidal choroidal vasculopathy (Ipcv). Retina 10:1-8

2. Yannuzzi LA, Ciardella A, Spaide RF, Rabb M, Freund KB, Orlock DA (1997) The expanding clinical spectrum of idiopathic polypoidal choroidal vasculopathy. Arch Ophthalmol 115:478-485

3. Balaratnasingam C, Lee WK, Koizumi H, Dansingani K, Inoue M, Freund KB (2016) Polypoidal choroidal vasculopathy: a distinct disease or manifestation of many? Retina 36:1-8. https://doi.org/ 10.1097/iae.0000000000000774

4. Dansingani KK, Gal-Or O, Sadda SR, Yannuzzi LA, Freund KB (2018) Understanding aneurysmal type 1 neovascularization (polypoidal choroidal vasculopathy): a lesson in the taxonomy of 'expanded spectra' - a review. Clin Exp Ophthalmol 46:189-200. https://doi.org/10.1111/ceo.13114

5. Spaide RF, Jaffe GJ, Sarraf D, Freund KB, Sadda SR, Staurenghi G, Waheed NK, Chakravarthy U, Rosenfeld PJ, Holz FG, Souied EH, Cohen SY, Querques G, Ohno-Matsui K, Boyer D, Gaudric A, Blodi B, Baumal CR, Li X, Coscas GJ, Brucker A, Singerman L, Luthert P, Schmitz-Valckenberg S, Schmidt-Erfurth U, Grossniklaus HE, Wilson DJ, Guymer R, Yannuzzi LA, Chew EY, Csaky K, Mones JM, Pauleikhoff D, Tadayoni R, Fujimoto J (2019) Consensus nomenclature for reporting neovascular agerelated macular degeneration Data: Consensus on Neovascular Age-Related Macular Degeneration Nomenclature Study Group. Ophthalmology. https://doi.org/10.1016/j.ophtha.2019.11.004

6. Cheung CMG, Lai TYY, Ruamviboonsuk P, Chen SJ, Chen Y, Freund KB, Gomi F, Koh AH, Lee WK, Wong TY (2018) Polypoidal choroidal vasculopathy: definition, pathogenesis, diagnosis, and management. Ophthalmology 125:708-724. https://doi. org/10.1016/j.ophtha.2017.11.019

7. Coscas G, Lupidi M, Coscas F, Benjelloun F, Zerbib J, Dirani A, Semoun O, Souied EH (2015) Toward a specific classification of polypoidal choroidal vasculopathy: idiopathic disease or subtype of age-related macular degeneration. Invest Ophthalmol Vis Sci 56: 3187-3195. https://doi.org/10.1167/iovs.14-16236 
8. Kokame GT, Liu K, Kokame KA, Kaneko KN, Omizo JN (2019) Clinical characteristics of polypoidal choroidal vasculopathy and anti-vascular endothelial growth factor treatment response in Caucasians. Ophthalmologica 10.1159(000503834):1-9. https:// doi.org/10.1159/000503834

9. Chang YC, Cheng CK (2019) Difference between pachychoroid and nonpachychoroid polypoidal choroidal vasculopathy and their response to anti-vascular endothelial growth factor therapy. Retina. https://doi.org/10.1097/iae.0000000000002583

10. Wong CW, Yanagi Y, Lee WK, Ogura Y, Yeo I, Wong TY, Cheung CM (2016) Age-related macular degeneration and polypoidal choroidal vasculopathy in Asians. Prog Retin Eye Res 53:107-139. https://doi.org/10.1016/j.preteyeres.2016.04.002

11. Freund KB, Zweifel SA, Engelbert M (2010) Do we need a new classification for choroidal neovascularization in age-related macular degeneration? Retina 30:1333-1349. https://doi.org/10.1097/ IAE.0b013e3181e7976b

12. Maruko I, Iida T, Saito M, Nagayama D, Saito K (2007) Clinical characteristics of exudative age-related macular degeneration in Japanese patients. Am J Ophthalmol 144:15-22. https://doi.org/ 10.1016/j.ajo.2007.03.047

13. Sho K, Takahashi K, Yamada H, Wada M, Nagai Y, Otsuji T, Nishikawa M, Mitsuma Y, Yamazaki Y, Matsumura M, Uyama M (2003) Polypoidal choroidal vasculopathy: incidence, demographic features, and clinical characteristics. Arch Ophthalmol 121:1392-1396. https://doi.org/10.1001/archopht.121.10.1392

14. Lafaut BA, Leys AM, Snyers B, Rasquin F, De Laey JJ (2000) Polypoidal choroidal vasculopathy in Caucasians. Graefes Arch Clin Exp Ophthalmol 238:752-759

15. Lorentzen TD, Subhi Y, Sorensen TL (2018) Prevalence of polypoidal choroidal vasculopathy in white patients with exudative age-related macular degeneration: systematic review and metaanalysis. Retina 38:2363-2371. https://doi.org/10.1097/iae. 0000000000001872

16. Kokame GT, deCarlo TE, Kaneko KN, Omizo JN, Lian R (2019) Anti-vascular endothelial growth factor resistance in exudative macular degeneration and polypoidal choroidal vasculopathy. Ophthalmol Retina 3:744-752. https://doi.org/10.1016/j.oret. 2019.04.018

17. Coscas G, Yamashiro K, Coscas F, De Benedetto U, Tsujikawa A, Miyake M, Gemmy Cheung CM, Wong TY, Yoshimura N (2014) Comparison of exudative age-related macular degeneration subtypes in Japanese and French patients: multicenter diagnosis with multimodal imaging. Am J Ophthalmol 158:309-318.e302. https:// doi.org/10.1016/j.ajo.2014.05.004

18. Bird AC, Bressler NM, Bressler SB, Chisholm IH, Coscas G, Davis MD, de Jong PT, Klaver CC, Klein BE, Klein R et al (1995) An international classification and grading system for age-related Maculopathy and age-related macular degeneration. The International Arm Epidemiological Study Group. Surv Ophthalmol 39:367-374

19. Chung WH, van Dijk EH, Mohabati D, Dijkman G, Yzer S, de Jong EK, Fauser S, Schlingemann RO, Hoyng CB, Boon CJ (2017) Neovascular age-related macular degeneration without drusen in the fellow eye: clinical spectrum and therapeutic outcome. Clin Ophthalmol 11:63-70. https://doi.org/10.2147/opth.s122568

20. Imamura Y, Engelbert M, Iida T, Freund KB, Yannuzzi LA (2010) Polypoidal choroidal vasculopathy: a review. Surv Ophthalmol 55: 501-515. https://doi.org/10.1016/j.survophthal.2010.03.004

21. Lip PL, Hope-Ross MW, Gibson JM (2000) Idiopathic polypoidal choroidal vasculopathy: a disease with diverse clinical spectrum and systemic associations. Eye (Lond) 14(Pt 5):695-700. https:// doi.org/10.1038/eye.2000.186

22. Davis SJ, Lauer AK, Flaxel CJ (2014) Polypoidal choroidal vasculopathy in white patients. Retina 34:2185-2191. https://doi.org/10. 1097/iae.0000000000000206
23. Agorogiannis EI, Pearce IA, Yadav S, Parry DG, Beare NAV (2018) Clinical outcomes in Caucasian patients with polypoidal choroidal vasculopathy. Eye (Lond) 32:1731-1739. https://doi. org/10.1038/s41433-018-0168-2

24. Koh A, Lee WK, Chen LJ, Chen SJ, Hashad Y, Kim H, Lai TY, Pilz S, Ruamviboonsuk P, Tokaji E, Weisberger A, Lim TH (2012) Everest study: efficacy and safety of verteporfin photodynamic therapy in combination with ranibizumab or alone versus ranibizumab monotherapy in patients with symptomatic macular polypoidal choroidal vasculopathy. Retina 32:1453-1464. https:// doi.org/10.1097/IAE.0b013e31824f91e8

25. Koh AH, Chen LJ, Chen SJ, Chen Y, Giridhar A, Iida T, Kim H, Yuk Yau Lai T, Lee WK, Li X, Han Lim T, Ruamviboonsuk P, Sharma T, Tang S, Yuzawa M (2013) Polypoidal choroidal vasculopathy: evidence-based guidelines for clinical diagnosis and treatment. Retina 33:686-716. https://doi.org/10.1097/IAE. 0b013e3182852446

26. Cheung CM, Lai TY, Chen SJ, Chong V, Lee WK, Htoon H, Ng WY, Ogura Y, Wong TY (2014) Understanding Indocyanine green angiography in polypoidal choroidal vasculopathy: the group experience with digital fundus photography and confocal scanning laser ophthalmoscopy. Retina 34:2397-2406. https://doi.org/10.1097/ iae.0000000000000255

27. Ojima Y, Hangai M, Sakamoto A, Tsujikawa A, Otani A, Tamura H, Yoshimura N (2009) Improved visualization of polypoidal choroidal vasculopathy lesions using spectral-domain optical coherence tomography. Retina 29:52-59. https://doi.org/10.1097/IAE. 0b013e3181884fbf

28. Sato T, Kishi S, Watanabe G, Matsumoto H, Mukai R (2007) Tomographic features of branching vascular networks in polypoidal choroidal vasculopathy. Retina 27:589-594. https:// doi.org/10.1097/01.iae.0000249386.63482.05

29. Cackett P, Wong D, Yeo I (2009) A classification system for polypoidal choroidal vasculopathy. Retina 29:187-191. https:// doi.org/10.1097/IAE.0b013e318188c839

30. Yuzawa M, Mori R, Kawamura A (2005) The origins of polypoidal choroidal vasculopathy. Br J Ophthalmol 89:602-607. https://doi. org/10.1136/bjo.2004.049296

31. Lehmann M, Bousquet E, Beydoun T, Behar-Cohen F (2015) Pachychoroid: an inherited condition? Retina 35:10-16. https:// doi.org/10.1097/iae.0000000000000287

32. Uyama M, Matsubara T, Fukushima I, Matsunaga H, Iwashita K, Nagai Y, Takahashi K (1999) Idiopathic polypoidal choroidal vasculopathy in Japanese patients. Arch Ophthalmol 117:1035-1042

33. Uyama M, Wada M, Nagai Y, Matsubara T, Matsunaga H, Fukushima I, Takahashi K, Matsumura M (2002) Polypoidal choroidal vasculopathy: natural history. Am J Ophthalmol 133:639648

34. Kwok AK, Lai TY, Chan CW, Neoh EL, Lam DS (2002) Polypoidal choroidal vasculopathy in Chinese patients. Br J Ophthalmol 86:892-897

35. Wen F, Chen C, Wu D, Li H (2004) Polypoidal choroidal vasculopathy in elderly Chinese patients. Graefes Arch Clin Exp Ophthalmol 242:625-629. https://doi.org/10.1007/s00417-0030667-z

36. Pereira FB, Veloso CE, Kokame GT, Nehemy MB (2015) Characteristics of neovascular age-related macular degeneration in Brazilian patients. Ophthalmologica 234:233-242. https://doi. org/10.1159/000439359

37. Augood CA, Vingerling JR, de Jong PT, Chakravarthy U, Seland J, Soubrane G, Tomazzoli L, Topouzis F, Bentham G, Rahu M, Vioque J, Young IS, Fletcher AE (2006) Prevalence of agerelated Maculopathy in older Europeans: the European Eye Study (Eureye). Arch Ophthalmol 124:529-535. https://doi.org/10.1001/ archopht.124.4.529 
38. Erke MG, Bertelsen G, Peto T, Sjolie AK, Lindekleiv H, Njolstad I (2012) Prevalence of age-related macular degeneration in elderly Caucasians: the Tromso Eye Study. Ophthalmology 119:17371743. https://doi.org/10.1016/j.ophtha.2012.03.016

39. Jung JJ, Chen CY, Mrejen S, Gallego-Pinazo R, Xu L, Marsiglia M, Boddu S, Freund KB (2014) The incidence of neovascular subtypes in newly diagnosed neovascular age-related macular degeneration. Am J Ophthalmol 158:769-779.e762. https://doi.org/10.1016/j.ajo. 2014.07.006

40. George S, Cooke C, Chakravarthy U (2010) Exudative Amd subtypes and eligibility for treatment with Ranibizumab. Eye (Lond) 24:1247-1251. https://doi.org/10.1038/eye.2009.301

41. Spaide RF (2018) Improving the age-related macular degeneration construct: a new classification system. Retina 38:891-899. https:// doi.org/10.1097/iae.0000000000001732

42. Ferris FL 3rd, Wilkinson CP, Bird A, Chakravarthy U, Chew E, Csaky K, Sadda SR (2013) Clinical classification of age-related macular degeneration. Ophthalmology 120:844-851. https://doi. org/10.1016/j.ophtha.2012.10.036

43. Cheung CMG, Lee WK, Koizumi H, Dansingani K, Lai TYY, Freund KB (2019) Pachychoroid disease. Eye (Lond) 33:14-33. https://doi.org/10.1038/s41433-018-0158-4

44. de Jong EK, Breukink MB, Schellevis RL, Bakker B, Mohr JK, Fauser S, Keunen JE, Hoyng CB, den Hollander AI, Boon CJ (2015) Chronic central serous chorioretinopathy is associated with genetic variants implicated in age-related macular degeneration. Ophthalmology 122:562-570. https://doi.org/10.1016/j.ophtha. 2014.09.026

45. van Dijk EHC, Tsonaka R, Klar-Mohamad N, Wouters D, de Vries APJ, de Jong EK, van Kooten C, Boon CJF (2017) Systemic complement activation in central serous chorioretinopathy. PLoS One 12:e0180312. https://doi.org/10.1371/journal.pone.0180312

46. Ma L, Li Z, Liu K, Rong SS, Brelen ME, Young AL, Kumaramanickavel G, Pang CP, Chen H, Chen LJ (2015) Association of genetic variants with polypoidal choroidal vasculopathy: a systematic review and updated meta-analysis. Ophthalmology 122:1854-1865. https://doi.org/10.1016/j.ophtha. 2015.05.012

47. Miki A, Honda S, Kondo N, Negi A (2013) The Association of agerelated maculopathy susceptibility 2 (Arms2) and complement factor $\mathrm{H}(\mathrm{Cfh})$ variants with two angiographic subtypes of polypoidal choroidal vasculopathy. Ophthalmic Genet 34:146-150. https://doi. org $/ 10.3109 / 13816810.2012 .749288$

48. Tanaka K, Nakayama T, Mori R, Sato N, Kawamura A, Mizutani Y, Yuzawa M (2011) Associations of complement factor H (Cfh) and age-related maculopathy susceptibility 2 (Arms2) genotypes with subtypes of polypoidal choroidal vasculopathy. Invest Ophthalmol Vis Sci 52:7441-7444. https://doi.org/10.1167/iovs. 11-7546

49. Laude A, Cackett PD, Vithana EN, Yeo IY, Wong D, Koh AH, Wong TY, Aung T (2010) Polypoidal choroidal vasculopathy and neovascular age-related macular degeneration: same or different disease? Prog Retin Eye Res 29:19-29. https://doi.org/10.1016/j. preteyeres.2009.10.001

50. Spaide RF, Fujimoto JG, Waheed NK (2015) Optical coherence tomography angiography. Retina 35:2161-2162. https://doi.org/ 10.1097/iae.0000000000000881

Publisher's note Springer Nature remains neutral with regard to jurisdictional claims in published maps and institutional affiliations. 